\title{
Symptomatic Posterior Cruciate Ganglion Cyst Causing Impingement between Posterior Root of the Medial Meniscus and Anterior to the Posterior Cruciate Ligament
}

\author{
Yong Bum Joo, MD and Young Mo Kim, MD, PhD \\ Department of Orthopedic Surgery, Chungnam National University Hospital, Chungnam National University College of Medicine, Daejeon, Korea
}

\begin{abstract}
There are several reports of symptomatic ganglion cysts near the posterior cruciate ligament (PCL), but no reports of a symptomatic ganglion originating from the anterior aspect of the PCL in the deep recess between the posterior root of the medial meniscus and the PCL, bulging into the posteromedial joint space. In this report, we present the clinical features of a patient with a symptomatic ganglion cyst treated successfully by arthroscopic excision.
\end{abstract}

Key words: Knee, Posterior cruciate ligament, Posterior root of medial meniscus, Ganglion cyst, Arthroscopic excision.

There are many reports of ganglion cysts observed in various joints. Cystic masses of the knee are relatively common ${ }^{1)}$, but intra-articular ganglion cysts related to the posterior cruciate ligament (PCL) of the knee are rare ${ }^{2,3}$. In particular, a cyst in the anterior aspect of the PCL is rarer than in the posterior aspect ${ }^{4)}$. We report a case of a symptomatic ganglion cyst that originated from the anterior aspect of the PCL in the deep recess between the posterior root of the medial meniscus (MM) and the PCL.

Received July 3, 2011; Revised (1st) August 2, 2011;

(2nd) September 7, 2011; Accepted September 15, 2011.

Correspondence to: Young Mo Kim, MD, PhD.

Department of Orthopaedic Surgery, Chungnam National University College of Medicine, 282 Munhwa-ro, Jung-gu, Daejeon 301-721, Korea. Tel: +82-42-220-7352, Fax: +82-42-252-7098

Email: osdr69@cnu.ac.kr

This is an Open Access article distributed under the terms of the Creative Commons Attribution Non-Commercial License (http://creativecommons.org/licenses/by-nc/3.0/) which permits unrestricted non-commercial use, distribution, and reproduction in any medium, provided the original work is properly cited.

\section{Case Report}

A 27-year-old male visited our hospital because of severe pain in the popliteal area that occurred when the knee was flexed actively beyond $100^{\circ}$ during squatting or flexed passively beyond $120^{\circ}$ in the crossed leg position. He had struck the lateral side of his left knee against the fence alongside a ski slope while snowboarding 8 weeks earlier, and the symptoms aggravated despite conservative management. He had no history of trauma prior to that event. On physical examination, the anterior drawer test was grade 1 (4 mm) and the Lachman test result was a firm end point. The pivot shift, posterior drawer and valgus stress tests were negative. The patient complained of tenderness around the medial femoral condyle (MFC).

Plain radiographs of the knee were normal. However, magnetic resonance imaging (MRI) of the left knee showed a bone marrow contusion on the lateral tibial plateau, sprains of the anterior cruciate ligament (ACL) and medial collateral ligament (MCL). In addition, MRI demonstrated a rounded and well-demarcated soft mass anterior to the PCL, and the ligament was slightly deformed due to the mass. The cystic lesion was seen originating from the deep recess between the posterior root of the MM and the PCL that bulged into the posteromedial joint space with a signal change in the posterior root of MM and the PCL adjacent 

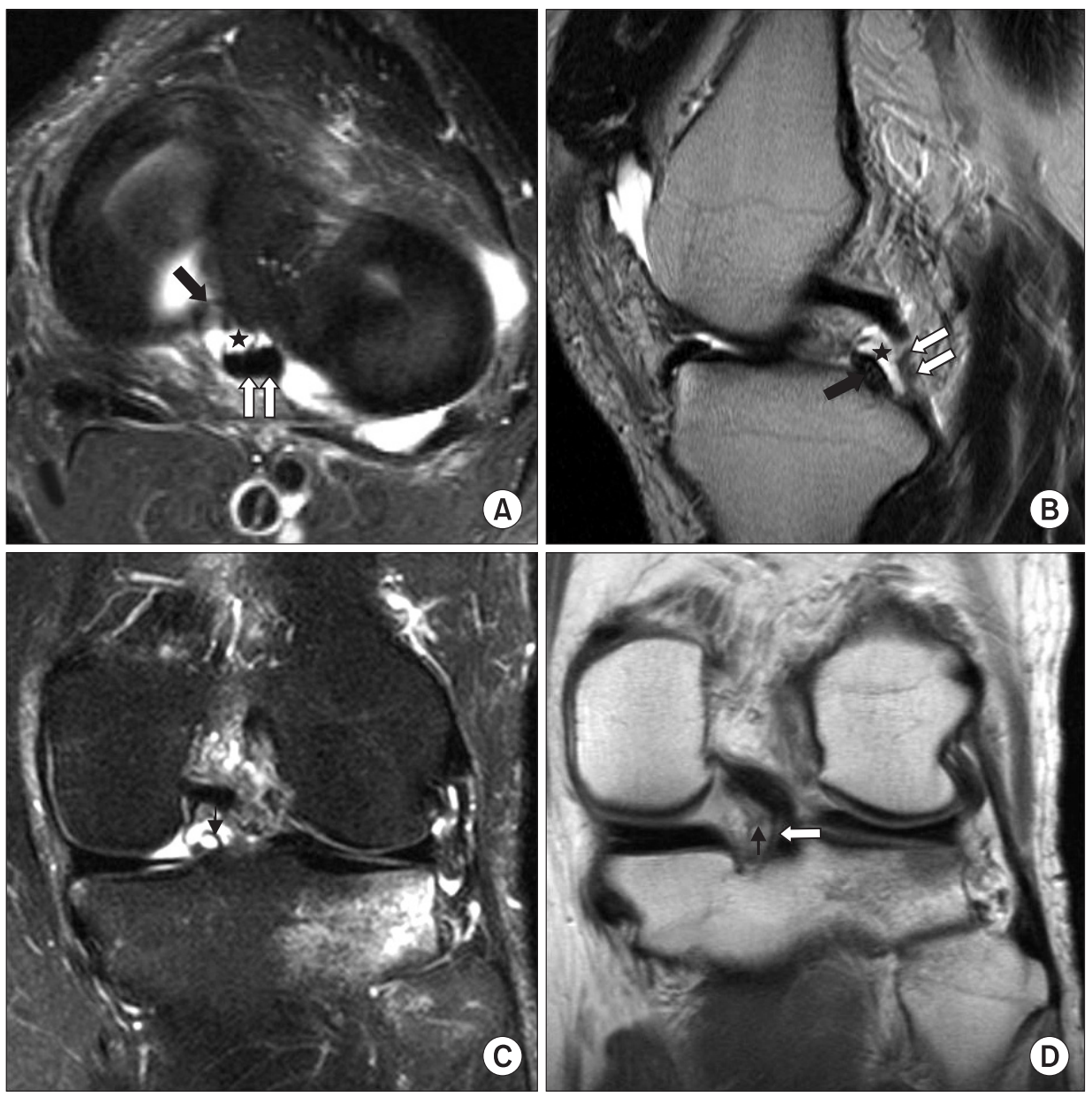

Fig. 1. (A) Axial fat-suppressed T2-weighted MR image and (B) sagittal turbo spin echo T2-weighted MR image showing an intervening lobulated cystic lesion (asterisk) located in the deep recess between the posterior root of the medial meniscus (black arrow) and the posterior cruciate ligament (PCL) (white arrows). (C) Consecutive coronal fat-suppressed T2-weighted MR images and (D) proton density MR image showing a lobulated cystic lesion with internal septation (small arrow) abutting the posterior medial femoral condyle. The cystic lesion abutted the anteromedial aspect of the PCL, which showed some fraying and increased signal intensity (white arrow).

to the cyst (Fig. 1).

Arthroscopy revealed a cystic lesion, about $7 \mathrm{~mm}$ in diameter, originating from the anterior aspect of the PCL between the posterior root of the (MM) and the PCL (Fig. 2A). During knee flexion, the cystic lesion enlarged as it was compressed between the posterior root of the MM and the PCL, and it impinged on the articular cartilage of the posterior MFC, the posterior root of the MM, and the PCL (Fig. 2B). In addition, a dimple was found on the articular cartilage of the posterior MFC adjacent to the cystic lesion (Fig. 2C). When the cyst was punctured, a jelly-like viscous fluid was extruded, and some degenerative soft tissue attached to the PCL was identified. We removed the cystic lesion completely along with some fibers from the PCL adjacent to the cystic lesion where the signal change was seen on MRI (Fig. 2D) and the specimen was sent to the pathology department. We did not find any significant instability related to the ACL, PCL, or MCL, and no other intra-articular pathology. The histologic examination of the cystic tissue showed proliferation of synovial cells with mild chronic inflammation and cystic space lined by fibrous connective tissue with focal myxoid degeneration, confirming the diagnosis of ganglion.

The symptoms improved immediately after the operation. At 12 months postoperatively, the patient was able to perform all activities of daily living, including squatting and sitting in the crossed leg position, and had full range of motion (ROM). No instability of the knee joint was detected. The Visual Analogue Scale had decreased from 9 preoperatively to 1 .

\section{Discussion}

Although ganglion cysts in various joints have been reported frequently, intra-articular ganglion cysts arising from the PCL are uncommon ${ }^{2,3)}$. Brown and Dandy ${ }^{5)}$ reported that only 6 of 35 ganglion cysts of the cruciate ligaments arose from the PCL in 6,500 arthroscopic examinations of knees over a period of 13 years. In addition, the cyst in the anterior aspect of the PCL is rarer than that in the posterior aspect ${ }^{4}$. In particular, we did not find any reports of a symptomatic ganglion originating from the anterior aspect of the PCL in the deep recess between the posterior root of the MM and the PCL that bulges into the 

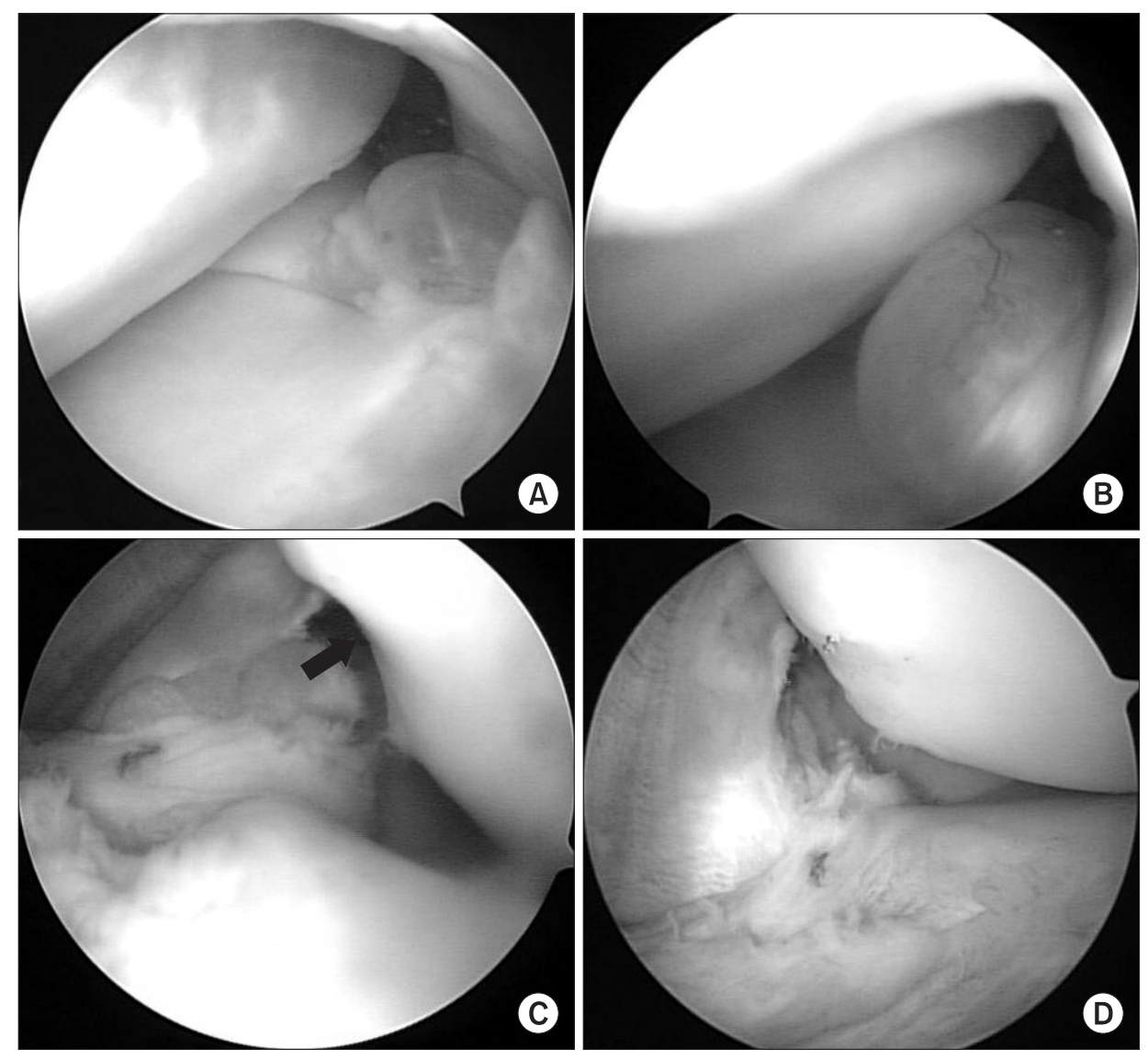

Fig. 2. Arthroscopic finding of the posteromedial compartment of the knee joint. (A) The ganglion cyst originated from the anterior aspect of the posterior cruciate ligament (PCL) in the deep recess between the posterior root of medial meniscus (MM) and the PCL. (B) During knee flexion, the cystic lesion was enlarged and impinged on the articular cartilage of posterior medial femoral condyle (MFC), the posterior root of the MM, and the PCL. (C) A dimple was found on the articular cartilage of the posterior portion of the MFC (arrow) where it was in contact with the cystic lesion at high flexion. (D) The cystic lesion and some fibers of the PCL adjacent to the cystic lesion were removed. posteromedial joint space.

Ganglion cysts arising from the PCL are increasingly recognized due to the sensitivity of MRI for visualizing intra-articular abnormalities. The finding of an ovoid fluid filled cystic lesion, which is frequently multilocular, in the intercondylar notch MRI has been described as characteristic ${ }^{3)}$. It has been suggested that these lesions should be treated by arthroscopic excision ${ }^{5}$. In our case, MRI showed the fluid filled and lobulated cystic lesion.

The clinical manifestations of a cyst are largely dependent on the pathologic process involved, along with its location, size, mass effect, and relationship to surrounding structures ${ }^{1)}$. One of the typical clinical manifestations seen with a ganglion cyst of the cruciate ligament is a diminished $\mathrm{ROM}^{2,6)}$. Tachibana et al. ${ }^{6}$ described the symptoms present with an intra-articular ganglion arising from the posterior joint capsule of the knee joint. They suggested that the development of knee pain during flexion and the inability to fully flex the knee could be explained by compression of the mass between the PCL and the posterior joint capsule. In our case, we believe that the pain arose from both compression of the ganglion cyst in the deep recess between the posterior root of the MM and the PCL during knee flexion, and the impingement of the ganglion cyst on the posterior MFC articular cartilage, the posterior root of the MM, and the PCL during flexion.

The histogenesis and pathogenesis of ganglia are unclear, and many theories have been proposed ${ }^{2}$. Trauma has been advocated as playing a role in the pathogenesis of ganglion formation ${ }^{2)}$. A blow to the lateral aspect of the leg mostly causes injuries to the medial side of the knee including the MCL, and may deliver a twisting force that can damage the ACL and possibly even the $\mathrm{PCL}^{7)}$. We postulated that the ganglion cyst that arose in our patient may be of traumatic origin based on the similarity between the MRI findings and the mechanism of injury.

Treatment options vary according to the symptoms and the suspected diagnosis in any given case ${ }^{1)}$. The complete resection of the mass is often technically demanding or impossible ${ }^{8)}$. We removed the ganglion cyst and some fibers of the PCL adjacent to the cystic lesion where a signal change was seen on MRI. Subsequently, the patient's symptoms disappeared.

\section{References}

1. Beall DP, Ly JQ, Wolff JD, Sweet CF, Kirby AB, Murphy MP, Webb H, Fish JR. Cystic masses of the knee: magnetic 
resonance imaging findings. Curr Probl Diagn Radiol. 2005;34:143-59.

2. Kim RS, Kim KT, Lee JY, Lee KY. Ganglion cysts of the posterior cruciate ligament. Arthroscopy. 2003;19:E36-40.

3. Coakley FV, Finlay DB, Harding ML, Oni OA. The nature and significance of cysts found at MR imaging behind the posterior cruciate ligament of the knee. Knee. 1996;3:139-43.

4. Bui-Mansfield LT, Youngberg RA. Intraarticular ganglia of the knee: prevalence, presentation, etiology, and management. AJR Am J Roentgenol. 1997;168:123-7.
5. Brown MF, Dandy DJ. Intra-articular ganglia in the knee. Arthroscopy. 1990;6:322-3.

6. Tachibana Y, Ninomiya T, Goto T, Yamazaki K, Ninomiya $\mathrm{S}$. Intra-articular ganglia arising from the posterior joint capsule of the knee. Arthroscopy. 2004;20 Suppl 2:54-9.

7. Azar FM. Surgical treatment of ACL/PCL/medial-side knee injuries. Oper Tech Sports Med. 2003;11:248-56.

8. Noda M, Kurosaka M, Maeno K, Mizuno K. Case report ganglion cysts of the bilateral cruciate ligaments. Arthroscopy. 1999;15:867-70. 